\title{
Muséologies
}

Les cahiers d'études supérieures

\section{Musées et virtualité : un univers de reconnaissance}

\section{Marie-Blanche Fourcade et Anik Meunier}

Volume 6, numéro 2, 2013

La cybermuséologie

URI : https://id.erudit.org/iderudit/1018926ar

DOI : https://doi.org/10.7202/1018926ar

Aller au sommaire du numéro

Éditeur(s)

Association Québécoise de Promotion des Recherches Étudiantes en

Muséologie (AQPREM)

ISSN

1718-5181 (imprimé)

1929-7815 (numérique)

Découvrir la revue

Citer ce document

Fourcade, M.-B. \& Meunier, A. (2013). Musées et virtualité : un univers de reconnaissance. Muséologies, 6(2), 8-13. https://doi.org/10.7202/1018926ar

Tous droits réservés (C Association Québécoise de Promotion des Recherches Étudiantes en Muséologie (AQPREM), 2013
Ce document est protégé par la loi sur le droit d'auteur. L’utilisation des services d'Érudit (y compris la reproduction) est assujettie à sa politique d'utilisation que vous pouvez consulter en ligne.

https://apropos.erudit.org/fr/usagers/politique-dutilisation/ 
Musées et virtualité: un univers de reconnaissance

Ce numéro thématique consacré aux relations que l'institution muséale et le monde numérique entretiennent trouve son origine dans un colloque qui s'est tenu en mai 2011, dans le cadre du $79^{\mathrm{e}}$ congrès de l'Association francophone pour le savoir (ACFAS). Intitulée Musée et virtualité: états des lieux d'une révolution annoncée, cette rencontre proposait à une douzaine de participants québécois issus des milieux professionnel-musées, associations, consultants, entreprises - et scientifique - professeurs, étudiants et chercheurs de l'Université du Québec à Montréal, de l'Université du Québec à Trois-Rivières, de l'Université Laval et de l'Université de Montréal - de se pencher sur la situation et l'avenir du projet virtuel, en marche depuis près d'une vingtaine d'années dans la sphère culturelle. Nous souhaitions plus précisément dans ce recueil nous interroger sur les résultats tangibles et intellectuels de la révolution numérique qui s'est déroulée au sein et en extension du musée.

Nous nous sommes donc permis de poser, sur cette course que l'on dit effrénée tout autant qu'enthousiasmante, un regard critique afin de comprendre, à partir des réussites, des échecs, des incertitudes et des projets en cours, comment le musée se développe dans la virtualité et, en retour, comment cette dernière, par imitation ou par opposition, tente de prendre une forme muséale.

En effet, il suffit d'explorer les transformations des fonctions muséales pour saisir les enjeux que pose la mise en place d'une plate-forme virtuelle. La conservation renvoie d'abord aux modalités de stockage, d'archivage et d'accessibilité aux données. L'exposition, quant à elle, amène à revoir les moyens de transmission et de partage de l'information. La recherche provoque une réflexion sur les temporalités convoquées entre le produit fini présenté et la construction théorique qui le sous-tend. La médiation, 
enfin, interroge l'expérience numérique dans son originalité et sa qualité, mais également dans ses conditions de fréquentation. Plus largement, les musées virtuels nous obligent à redéfinir notre rencontre avec les musealia et les lieux de mise en valeur selon des variables de temps et d'espace renouvelées. Face au foisonnement des formes que recouvre l'appellation du musée virtuel, pouvons-nous esquisser son modèle générique, pouvonsnous également justifier sa nécessité et sa légitimité?

En somme, il s'agit d'un échange non seulement sur les défis lancés par l'expérience virtuelle à la muséologie et à ceux que la discipline pose en retour au cyberespace, mais également sur les transformations qui sont en train de s'opérer au cour de nos pratiques et des valeurs qui ont depuis l'origine régi le musée. Dans l'idée d'un partage qui rassemble tant les cas d'expériences institutionnelles que des propositions théoriques, nous avons ainsi tenté d'éclairer avec justesse la complexité qui se cache dans l'ombre de l'utopie numérique.

Afin de poursuivre et d'approfondir la réflexion amorcée, notre numéro propose, à partir de cas d'études et d'expériences de terrain, plusieurs analyses autour de pratiques muséales dans le cyberespace et d'usages du virtuel au musée. Marc Terrisse ouvre la discussion en s'intéressant à l'évolution de la visite en ligne, des années 1990 à nos jours, soit à partir de l'arrivée des premiers cédéroms réunissant quelques fragments des grandes collections muséales jusqu'à la visite en ligne proposée par le Google Art Project. Dans une perspective critique, l'auteur met en dialogue la récente histoire des pratiques avec les courants d'idées qui ont marqué les différentes étapes d'appropriation du Web. Engouement, craintes, comparaisons se croisent ainsi au profit d'un essai de définition qui nous permet de voir avec davantage de clarté 
certains défis et enjeux que pose cette forme muséale et d'interroger, pour aller plus loin, les effets du virtuel non plus seulement sur les structures nées numériques, mais également sur les visiteurs qui en profitent.

Dans un même esprit, Marie-Blanche Fourcade porte attention, par l'entremise de trois projets de médiation de témoignages en ligne-Dane Wajich. Contes et chants: les rêveurs de leur terre (2007), le Musée de Toronto en ligne (2011) et Sacrée montagne (2011) -, à l'expérience patrimoniale numérique. À travers cette étude qui permet d'explorer différentes modalités de cybervisite, l'auteure nous invite à analyser les possibles effets du numérique sur le patrimoine en contexte de mise en valeur et, par le fait même, sur les fonctions, les pratiques et les valeurs qui fondent l'institution muséale. S'il est certain que de nouveaux codes ou une nouvelle grammaire sont nécessaires aux visiteurs pour aborder le patrimoine en ligne, les interactions qui sont provoquées offrent, également, des avenues alternatives d'interprétation, de partage et de transmission qui façonnent elles-mêmes un autre regard sur le patrimoine et la patrimonialisation.

Avec Dominique Gélinas, nous entrons dans le quotidien de l'institution muséale et, plus précisément au cœur du processus de création expographique. En partant du constat d'une intégration numérique réussie mais néanmoins inachevée en raison, entre autres, de moyens techniques et de stratégies de médiation de contenu qui n'ont pas encore atteint leur plein épanouissement, l'auteure nous propose de tracer à grands traits les ingrédients du succès dans la création d'un multimédia. Au-delà des questions techniques, Dominique Gélinas présente les transformations qu'apportent de telles productions, au niveau tant de l'expérience des visiteurs que de la démarche des créateurs. Elle soulève également 
avec pertinence plusieurs questions concernant le sens de l'intervention multimédia, questions qui devraient constituer le point de départ de la démarche expographique : quels sont les véritables besoins du public? Comment rendre le projet numérique distinctif? En guise de réponse, des mots-clés surgissent : collaboration, entraide et expérimentation.

Walter Guyot, Hélène M. Courtois et Jacques Toussaint nous entraînent dans une virtualité appliquée, cette fois, au domaine de la muséologie scientifique. Les auteurs nous montrent ainsi de quelle manière le numérique se fait au sein des planétariums - et plus particulièrement dans celui de Vaux-en-Velin au centre de l'analyse - et devient un outil incontournable. Les atouts énoncés par ce qu'ils nomment "de véritables environnements virtuels d'apprentissage humain " touchent plusieurs aspects de la vie muséale, notamment les pratiques d'acquisition et de conservation ainsi que les processus de médiation du patrimoine cosmographique. La production et l'archivage de données d'observation, d'une part, et leur mise en accès par le biais d'interactions multisensorielles avec le public, d'autre part, permettent aux visiteurs de transcender la cristallisation des artefacts dans le temps propre au monde muséal au profit d'une matière active logée entre la recherche et la mise en valeur.

S'ajoutent aux quatre articles présentés deux entrevues qui permettent de croiser les regards de deux acteurs du monde muséal sur la relation qu'entretiennent les institutions et le numérique. L'entrevue de Bernard Deloche, philosophe et muséologue, rappelle que la concurrence entre musées et nouveaux médias renvoie à la question de la nature de l'action exercée. Cette action induit de nouveaux médias qui produisent et diffusent une nouvelle culture. Il propose ainsi trois modèles (identitaires, 
comportementaux et mentaux) qui témoignent du changement de la culture au sens fort. L'enchevêtrement de ces modèles sociaux atteste en quelque sorte la redéfinition de la culture, mais caractérise aussi la culture actuelle. S'ensuit une concurrence affirmée pour le musée et les autres médias qui l'entourent. Puis, les questions de la diversification de la médiation, du développement de différentes catégories de publics et de la formation des étudiants en muséologie sont abordées, toujours en lien avec les nouvelles technologies qui s'imposent de plus en plus dans les différentes sphères d'activité et d'action du musée. Bernard Deloche nous guide au cour d'une réflexion perspicace et combien pertinente pour tenter de comprendre l'inscription de la portée du musée dans le cyberespace.

Monique Camirand nous propose en entrevue un regard réflexif sur une riche pratique professionnelle dans les musées et les expositions mettant à contribution des technologies numériques. En effet, elle pose sur différents projets de médiation, d'animation et d'éducation muséale des constats qui permettent une certaine analyse de l'intégration des dispositifs technologiques de différentes natures. De plus, son propos étaye et développe des voies afin d'envisager les effets éducatifs dont peuvent bénéficier les visiteurs et les usagers de ces contextes technologiques. Elle aborde des exemples de succès et d'échecs de l'intégration de nouvelles technologies dans les musées. Puis, elle soulève les enjeux de la conception des contenus numériques dans les expositions et les programmes éducatifs. Enfin, elle remarque que les usages que les technologies induisent sont peut-être déjà bien présents dans les pratiques hors les murs des visiteurs et que ces derniers les intègrent tout simplement à leurs modes de visites des musées et des expositions. 
Enfin, en hors-thème de ce numéro, Alice Chatzimanassis présente un texte qui porte sur une évaluation conduite au musée d'Ixelles, à Bruxelles. Comme ce dernier prévoyait actualiser le dispositif didactique de ses collections permanentes, Alice Chatzimanassis a procédé, dans le cadre d'un travail dirigé, à une démarche empirique proposant une évaluation des textes d'exposition. Il s'est agit d'observer, d'identifier et de comprendre les modes d'appropriation et le comportement des visiteurs envers le dispositif textuel dans deux expositions en cours au musée d'Ixelles au mois d'avril 2010, à savoir EL CUBISMO. Cubism and its Context from the Fundación Telefónica Art Collection et Henri de Toulouse-Lautrec: La collection complète des affiches. Si l'importance du texte en tant qu'aide à l'interprétation dans les musées est aujourd'hui largement reconnue, son enquête qualitative a permis de développer une réflexion sur les spécificités de l'écrit quand il est confronté à des œuvres d'art et sur ses fonctions dans la pratique de contemplation et d'interprétation chez les visiteurs. 